http://dx.doi.org/10.18778/2196-8403.2007.06

JUSTYNA GÓRNY

\title{
Von der Novellistik zur Equilibristik. Literaturkriti- sche Wahrnehmung der Prosa von Franziska zu Reventlow von ihrem Debüt als Romanautorin 1904 bis 1927
}

W krytyce literackiej końca XIX w. literacka twórczość kobiet odczytywana była najczęściej w kontekście ich płci, co oznacza, że w omówieniach ich tekstów posługiwano się pojęciem ,kobiecości ${ }^{`}$. Nie jest to jednak zawsze równoznaczne z negatywnym przyjęciem tekstów kobiet. Również recenzenci prozy Franziski zu Reventlow (18711918) starali się stworzyć jej pozytywną interpretację za pomocą pojęcia ,kobiecości‘. Marginalizujące oddziaływanie takiego spojrzenia na tekst uwidacznia się dopiero z dystansu: skupienie na ,kobiecości ‘ autorki spowodowało, że w recenzjach nie wypracowano żadnego wzorca interpretacji prozy Reventlow, który nadawałby się do przejęcia lub zdekonstruowania przez literaturoznawstwo. Ponadto ciagłe wskazywanie na płeć autorki sprawiło, że zainteresowanie badaczy i badaczek skupiło się na aspekcie biograficznym. Biografia z kolei postrzegana była nie na przykład jako surowy materiał dla prozy, lecz jako jej temat i istota.

In der Kritik und in der Literaturwissenschaft des ausgehenden 19. Jh. wurde das schriftstellerische Schaffen von Frauen meistens im Zusammenhang mit ihrem Geschlecht gelesen, was bedeutet, dass in den Besprechungen ihrer Texte die ,Weiblichkeit" problematisiert wurde. Es bedeutet aber nicht immer, dass die Literaturkritik die Texte von Frauen dadurch abwerten wollte. Auch die Rezensenten der Prosa von Franziska zu Reventlow (1871-1918) waren bemüht, eine positive Interpretation, die die ,Weiblichkeit‘ einbezieht, zu entwickeln. Die ausgrenzende Potenz dieser Betrachtungsweise wird erst aus dem zeitlichen Abstand bemerkbar: Die Fixierung auf die ,Weiblichkeit' der Autorin hatte zur Folge, dass in den Rezensionen keine Interpretationsmuster für Reventlows Texte ausgearbeitet wurden, die dann durch die literaturwissenschaftliche Forschung hätten übernommen oder auch dekonstruiert werden können. Außerdem wurde durch das ständige Hinweisen auf das Geschlecht der Autorin der 
Justyna Górny

Schwerpunkt des Interesses auf den biographischen Aspekt verlagert. Die Biographie wiederum wurde nicht etwa als Rohmaterial für die Prosa, sondern als deren Inhalt und Wesen aufgefasst.

Literary criticism of the late 19th century analysed the women's literary works in the context of their gender, which means that the reviewers used the term ,femininity in relation to these works. This is not to say that their works were always given a negative response. The reviewers of the prose of Franziska zu Reventlow (1871-1918) tried to create a positive interpretation supplying it with the term ,femininity'. It is only when viewed from a distance that the marginalizing influence of that approach becomes visible: the reviews, while being quick to underline the ,femininity " of the author, were unable to present any pattern of interpretation of the prose of Reventlow that the academic literary criticism could make use of or deconstruct. Moreover, constant reminding of the author's sex made the male and female researchers focus on the biographical aspect. This biography, in turn, was not treated - for instance - as a rough basis for the prose, but as its subject and substance.

In der Kritik und in der Literaturwissenschaft des 19. Jahrhunderts wurde schriftstellerisches Schaffen von Frauen meistens im Zusammenhang mit ihrer Biographie und vor allem mit ihrem Geschlecht gelesen, was bedeutet, dass in den Besprechungen ihrer Texte die ,Weiblichkeit' problematisiert wurde. Es wurden z.B. bestimmte, ,typisch weibliche' Fehler bemerkt, aber auch bestimmte Gattungen wie Brief oder Tagebuch als , weiblich“ markiert, als literarische Gattungen, die sozusagen der täglichen Erlebniswelt der Frauen angehörten. ${ }^{1}$ Daher ernteten die Schriftstellerinnen das Lob der Kritik für die Stellen in ihren Texten, die fiktive Briefe und Tagebucheintragungen waren, wie das auch in den Aufsätzen über den Roman Florentin von Dorothea Schlegel der Fall war. Florentin erschien 1801, da aber die Autorin als Partnerin von Friedrich Schlegel und als eine Person aus dem Jenaer Kreis das Interesse der

1 Barbara Hahn zitiert aus einem Brief Wilhelm von Humboldts an seine Verlobte: „Sie brauchen nur aus Ihrer Seele abzuschreiben“, schreibt Humboldt und meint damit, dass der Brief seiner Geliebten so schön und interessant sei. Hahn interpretiert diese Bemerkung wie folgt: Es gebe einen Gegensatz zwischen dem reflektierenden Denken und der „Herzensschrift“. Die Sprache des Herzens sei das Attribut der Frauen, und ihre Briefe seien so reizend, weil sie von der Reflexion nicht verunreinigt würden. Man könnte auch hinzufügen, dass diese Sprache des Herzens, im Gegensatz zur Reflexion, natürlich sei, denn die Frauen seien der Natur näher als die Männer. So hätten sie in ihren Briefen die Reinheit der Sprache und die Unmittelbarkeit der Schilderung behalten können. Auch andere Faktoren könnten hierzu genannt werden. Man könnte z.B. behaupten, Briefschreiben sei Frauen ,erlaubt", weil diese Beschäftigung die Sphäre des Privaten nicht überschreitet. Mehr dazu in BECKER-CANTARINO (1985) und in HAHN (1988). 
Forschung weckte, kann man Besprechungen ihrer Texte sowohl aus dem ganzen 19. als auch vom Anfang des 20. Jahrhunderts finden. Dadurch erscheint die Rezeption ihrer Prosa als gutes Beispiel für die Wahrnehmung der Texte von Frauen in diesem Zeitraum. So gaben die Forscherinnen und Forscher, die sich mit ihrem Text beschäftigten, gewöhnlich zu, dass sie sehr schöne Briefe in den Roman ,eingestreut“ habe, wobei sie den restlichen Romantext als Ergebnis der ,geschickten Nachahmung“ anderer Autoren und als Beweis für ihre ausgesprochen weibliche Gabe der genialen Kompilation einschätzen. ${ }^{2}$ Die literaturwissenschaftliche Rezeption des Romans von Schlegel ist kein Sonderfall, die Texte von Frauen wurden in der Regel nicht in den Kontext der Literatur platziert, sondern vor dem Hintergrund des Geschlechts der Autorin gelesen. ${ }^{3}$ Die Vermutung liegt nahe, dass durch diese Herangehensweise an die Texte der Zugang zu ihnen weitgehend versperrt wird und dass die Rezeption infolge der mangelnden Einbindung in literarische Kontexte nicht zur Aufnahme in den literarischen Kanon führen konnte (HEYDEBRAND 1998). Daher erscheint es in Bezug auf die Texte von schreibenden Frauen im 19. und beginnenden 20. Jahrhundert besonders sinnvoll, ihre Rezeption unter dem Gender-Gesichtspunkt zu betrachten. Man muss dabei berücksichtigen, dass die von der Kritik betriebene ,Lektüre im Kontext der Weiblichkeit` keineswegs eine normierte Vorgehensweise war, was u.a. bedeutet, dass sich die Bezüge zu ,Weiblichkeit' sehr unterschiedlich auf die Wahrnehmung der rezensierten Texte auswirken konnten - dies musste nicht immer zur unmittelbaren negativen Bewertung des Textes führen! Darüber hinaus bilden die Rezensionen einen Teil des Weiblichkeitsdiskurses, es gab kein externes einheitliches Weiblichkeitskonzept, auf das sie sich beziehen würden. Der Weiblichkeitsdiskurs

2 BRAHM (1915:13) stellt fest, Schlegels Prosa zeichne sich durch ,,[...] eine natürliche Bildlichkeit aus, die sich mit Vorliebe aus dem Bereich der Küche ihre Anregung holt“. Die Anerkennung der Forschung fanden auch ihre „Genreszenen“ (DEIBEL 1905:53) und die ,humoristisch-realistischen Szenen“ (НAYM 1870:729). Schlegel wird in der Forschung als eine Schriftstellerin eingeschätzt, die recht begabt ist, wenn sie über die der Frau in ihrer täglichen Erfahrung zugänglichen Dinge schreibt, über die Szenen, die in jeder Gesellschaft stattfinden könnten, wie die von Haym gelobte Beschreibung des Hochzeitstages.

3 Daher die bei jeder schreibenden Frau aufkommende Frage, ob sie der Frauenliteratur angehöre oder nicht. Auch im Falle von Schriftstellerinnen wie Marie von Ebner-Eschenbach, Annette von Droste-Hülshoff oder Else Lasker-Schüler, die in weiterer Rezeption nicht zur (wie auch immer definierten) ,Frauenliteratur' gezählt werden, wurde in den ersten kritischen Besprechungen diese Frage nach den Bezügen zwischen Text, Weiblichkeit und Frauenliteratur gestellt und erörtert. 
Justyna Górny

selbst war um die Jahrhundertwende sehr komplex. Es existierten zwar Motive oder Formulierungen über, Weiblichkeit", die anerkannter waren, trotzdem war der Weiblichkeitsdiskurs eine differenzierte Erscheinung. ${ }^{4}$ Die ,Weiblichkeit" eines Textes wurde nicht ausschließlich als ein negatives Merkmal aufgefasst. Sehr oft war ,Weiblichkeit' nicht (nur) das Mittel, den Text einer Frau als minderwertig abzutun, sondern eine gedankliche Kategorie, mit der die Kritik versuchte, einen bestimmten Text zu erschließen. Wobei man wiederum beachten muss, dass sie dann wie jede Kategorie in unterschiedlichem Grad reflektiert wurde: Manche Kritiker gehen mit der Kategorie ,Weiblichkeit sehr bewusst um, für andere bleibt sie ein Begriff, den sie intellektuell konformistisch benutzen, ohne viel über ihn nachzudenken. Eine andere Frage ist allerdings, wie sich diese (wie auch immer gestaltete) Kontextualisierung der Texte von Frauen als ,Weiblichkeitsdokumente' auf die Rezeption des jeweiligen Textes und der Autorin im Ganzen auswirkte. Dieser Frage möchte ich exemplarisch nachgehen und mich der literaturkritischen Wahrnehmung der Prosa von Franziska zu Reventlow unter dem Gender-Aspekt analytisch annähern.

Franziska zu Reventlow gehört zwar nicht zum germanistischen Kanon, ist aber auch keine vergessene Schriftstellerin, vor kurzem erschien sogar die Ausgabe ihrer gesammelten Werke (REVENTLOW 2004). In der Nachkriegszeit war es aber ihr - tatsächlich interessantes - Leben, das das Interesse der Forschung weckte, wobei ihre literarischen Texte als autobiographische Zeugnisse gelesen wurden (FRITZ 1980). Seit den 60er Jahren wird ihrem Lebenslauf immer mehr Aufmerksamkeit geschenkt, zum einen weil sie ein Teil der Münchener Bohème war, zum anderen im Zuge des lokalen Interesses der Husumer Landsleute an ihr. Der Lebenslauf von Reventlow scheint auch eine gute Begründung für diese Perspektive zu sein. Die 1871 geborene Gräfin verzichtete auf ein standesgemäßes Leben und ging nach München, um Malerei zu studie-

$4 \quad$ So wurden die Frauen zum einen als asexuell, zum anderen aber als exzessiv sexuell aufgefasst. Mutterschaft wurde als Instinkt betrachtet, aber gleichzeitig verlangte man nach einer Erziehung der Mädchen, die darauf ausgerichtet wäre, dass sie die Mutterschaft in vollen Zügen verwirklichen könnten. Die Frau wurde als dem Mann geistig und körperlich untergeben betrachtet, was begründen sollte, dass die Frauen nicht beruflich arbeiteten. Andererseits pries man die unberührte Heiligkeit des Hauses, die nur von einer Frau erhalten werden könne und ohne die der Mann nicht überleben könne. Die Frau konnte als ein zutiefst religiöses oder aber als ein amoralisches Wesen betrachtet werden. Die Beispiele könnte man mehren, denn das Konzept der Weiblichkeit war eine dynamische gedankliche Konstruktion, die in verschiedenen Bezügen zu Normen des Alltags stand. 
ren. Dort verkehrte sie in den Kreisen der Bohème, war u. a. eine Zeitlang mit Ludwig Klages, Mitglied der „Kosmikerrunde“, befreundet. Sie unterhielt sich und ihren unehelichen Sohn Rolf durch unterschiedliche Tätigkeiten, u.a. übersetzte sie viel aus dem Französischen. Außer Romanen schrieb sie auch Erzählungen, Aufsätze und Kurzgeschichten, die u. a. im Simplicissimus und in den Züricher Diskußionen von Oskar Panizza veröffentlicht wurden. Reventlow starb 1918 in Ascona. ${ }^{5}$ Im Unterschied zu ihrer Biographie wurde ihre Prosa im Laufe der Zeit entdeckungsbedürftig - diese Entdeckung erfolgte dann mindestens teilweise durch die feministisch inspirierte Forschung. ${ }^{6}$ Es gibt also eine Disproportion: Der Biographie, als Leben einer Frau der Bohème begriffen, wird viel mehr Aufmerksamkeit geschenkt als der Prosa. Ein Ausblick auf die frühe Rezeption wirft vielleicht etwas Licht auf die möglichen Ursachen dieser Disproportion.

Die oben skizzierte Arbeitsthese, die Texte von Frauen seien stets im Kontext der Biographie unter besonderer Berücksichtigung des Geschlechts gelesen worden, macht die Lektüre der Rezensionen zum ersten Roman von Reventlow zu einem geradezu verblüffenden Erlebnis. Denn die stark autobiographisch unterfütterte Geschichte von Ellen Olestjerne wurde von der Kritik nahezu ohne Rückgriffe auf die Person der Autorin gelesen. Das Interesse an Reventlow selbst geht in diesen Rezensionen nicht über kurz angedeutete Vermutungen, dass es sich bei dem Roman vielleicht um Erlebnisse der Autorin handle, hinaus. Diese Feststellungen werden aber nur ganz am Rande gemacht und wirken sich auf das Verständnis des Textes von Reventlow nicht aus. Die Protagonistin, Ellen Olestjerne, wird zwar wie eine lebendige Person behandelt, wodurch das Buch zu einer Biographie wird. Das war aber eine übliche Vorgehensweise der Kritik, was auch bei Texten von Männern zum Vorschein kommt. Besonders wichtig zu sein scheint allerdings, dass diese ,lebendige Person“ Ellen nicht mit Franziska zu Reventlow gleichgesetzt wird - fiktive Biographie wird nicht zur, realen ' Autobiographie. Einer der Gründe für dieses Desinteresse an der Biographie mag sein, dass Ellen Olestjerne Reventlows Debüt und die Autorin selbst noch unbekannt war. Da die Kritik nicht über biographisches Wissen verfügte, konzentrierte sie sich auf den Text selbst. Und so wurde der Roman als die Darstellung eines für eine ganze Generation typischen Lebenslaufs aufgefasst, was ihm eine gewisse Universalität verlieh

Vgl. die grundlegende Monographie von KUBITSCHEK (1998).

6 Außer SzÉKELY (1979), der nicht durch die Gender-Forschung inspiriert war, sind es z.B. Jost (2002), KaloyANOva-SLAvova (1998), EdEN (1998) u. a. 
Justyna Górny

und vielleicht verhinderte, dass er etwa als ein individueller Entwurf einer Frau gelesen wurde. Es gehe hier, so die Kritik, um die Generation, die in den 80er Jahren des 19. Jahrhunderts im Namen Nietzsches und Ibsens gegen die alten und gegen die herkömmlichen Traditionen rebelliert habe. So wird Ellen Olestjerne in der Rezension von ST.[?] (1904:765-767), die in der Neuen Rundschau veröffentlicht wurde, verstanden. Auf die Charakteristik des Jugend-Aufstandes der 80er Jahre folgt die Feststellung: „Ellen Olestjerne ist in allen Lebensäußerungen eine typische Vertreterin jener stürmischen Zeit. [...] Aber das Buch gibt unendlich viel mehr als einen typischen Fall; es lässt sich keine Konstellation ersinnen, wo jener Gegensatz der Generationen zu stürmischeren Wendungen des Lebens, zu härteren Konflikten hätte führen können.“ Die Geschichte von Ellen Olestjerne ist also nicht nur „Geschichte eines Lebens“, wie der Untertitel des Romans ankündigt. Sie wird in der Rezension als eine synthetische Darstellung der besagten Generation aufgefasst, was auf die überindividuelle Ebene verweist. Anscheinend ist diese Synthese so dargestellt, dass sich mit ihr die einzelnen Mitglieder dieser Generation identifizieren konnten, zu der sich auch der Autor/die Autorin des Beitrags zählte:

Vor mir liegt ein Buch, das den Reiz und Zauber jener ersten anti-konventionellen Schilderhebung mir ganz zurückgerufen hat. Ellen Olestjerne, eine Lebensgeschichte von F. Gräfin zu Reventlow. Nicht so sehr in die Ideen, als in die Grundstimmung jener Tage führt uns die Verfasserin zurück. Sie ist zu frauenhaft, um im Intellektuellen ihre Stärke zu haben; Theoretisieren liegt ihr fern: sie erzählt uns ein Schicksal. (ST.[?] 1904:766)

Auch der Titel der Rezension deutet den überindividuellen Kontext an: Das Buch einer Zeitströmung. Vielleicht verursacht gerade diese Kontextualisierung des Romans, dass die Bezüge zu der realen Person Franziska zu Reventlow bei der Interpretation des Romans ohne Belang sind. Das schließt keineswegs aus, dass das Interesse an dem Buch vor allem das Interesse an der Protagonistin und ihrer Biographie ist. Ellen Olestjerne wird wie eine reale Person behandelt, wodurch die Besprechung des Romans stark psychologisiert wird. So schreibt z. B. HÄNNY SIMONS-STÖCKER (1906:26) über Ellen im Mutterschutz:

Um der Heldin gerecht zu werden, muß man sie nur als den lebenshungrigen, impulsiven Menschen ansehen, der trotz allen Unglücks, aller Enttäuschung genug zähe Kraft besitzt, sich durchzusetzen. Sie erkennt die ausgetretenen Pfade der Sitte, der althergebrachten Moral nicht an, sie durchlebt nicht viel innerliche Konflikte, aber sie trägt ohne Murren die Konsequenzen ihrer Handlungsweise. 
Ellen Olestjerne ist eine Person, deren Verhalten nach denselben Maßstäben wie das der realen Menschen beurteilt werden kann. Besonders deutlich kommt dies im Zusammenhang mit der Mutterschaft zum Ausdruck: SimonsStöcker bezweifelt, ob Ellen der Mutterschaft unter solch schwierigen Bedingungen gewachsen sei. ${ }^{7}$ In der Rezension von SIMONS-STÖCKER (1906:26) befindet sich eine Stelle, die ein interessanter Kommentar für diese Behandlung von Ellen als lebendige Person ist. „Das Buch macht den Eindruck des wirklich Erlebten, und man hat gleich von Anfang an ein starkes, steigendes Interesse für die Titelheldin. Sie ist gut gezeichnet, voller Leben und durch und durch echt. Hinter Ellen tritt alles andere zurück.“ Ellen findet Anerkennung als eine literarische Figur, die so ,gut gezeichnet ist“, dass sie ,voller Leben“ erscheint. „Das Buch“ ist zwar nicht die Beschreibung eines Lebens, macht aber „,den Eindruck“, als ob es das wäre. Es wird also von einem literarischen Text ausgegangen, und eben vom Text aus gelangt man zur Beurteilung der psychologischen Wahrscheinlichkeit dieser literarischen Figur - einer Figur, die mit der Autorin nicht gleichgesetzt wird.

Auch in der Rezension von Rainer Maria Rilke kommt keine Gleichsetzung der Figur Ellen mit der Autorin zustande, obwohl diese Rezension in der Form eines Briefes (Franziska Gräfin zu Reventlow, Ellen Olestjerne) an Ellen geschrieben ist, wodurch sich die Psychologisierung der Figur bis hin zu deren Belebung steigert. Der Brief ist ,real', weil er an der Stelle publiziert ist, die für nicht fiktive Äußerungen vorgesehen ist. Durch die ,Realität' des Briefes gewinnt auch Ellen an ,Realität‘. Es ist aber immer nur Ellen, nicht Ellen-Reventlow: „Liebe Ellen Olestjerne, nun hat man Ihre Geschichte erzählt; und ich finde das gut. [...] ich glaube, daß man es vor allem jungen Menschen erzählen muß, jungen Mädchen und jungen Männern, die das Leben anfangen wollen und nicht wissen, wie.“ (Zit. nach KUBITSCHEK 1998:565) Rilke unterscheidet Ellen von einer Instanz - es ist nicht einmal eine Person - die ihre Geschichte erzählt hat. „Man“ hat ihre Geschichte erzählt - und nun ist diese Geschichte

\footnotetext{
Eine besonders einfühlsame Psychologisierung in Bezug auf die Mutterschaft gibt es in der Rezension aus der Frankfurter Zeitung: „Sie fühlt sich Mutter werden von dem Manne, den sie liebt, - sie, die nach München kam, um der Kunst zu dienen, und die kaum für sich selbst das nötige Brot zum Leben hat. Wie eine Somnambule geht sie umher, getragen von der Kraft ihres inneren Erlebens. Sie ahnt, daß ein großes Geheimnis sich in ihr vollzieht, ein Geheimnis, das sie vor dem größten Manne voraus hat: selbst am Werden der Natur mitzuhelfen, Erdboden zu sein für eine neue Menschenpflanze. Mit unsäglichem Mutterschmerz und mit Todesverzweiflung lernt sie das Martyrium des Weibes begreifen.“ (S. [?] 1904:1f.)
} 
Justyna Górny

an sich wichtig. Auch Rilke unterstreicht die Universalität von Ellens Geschichte. Diesmal geht es nicht darum, dass sie für eine frühere Generation repräsentativ sei, sondern darum, dass sie für die jetzige junge Generation repräsentativ sein könne. Ellen Olestjerne wurde als eine erzählte Lebensgeschichte gelesen. Diese Lebensgeschichte wurde als ein literarischer Text behandelt, den es nach dem Kriterium der psychologischen Wahrscheinlichkeit zu beurteilen gilt. Über Ellen wird wie über eine lebendige Person gesprochen, es werden aber keine Verknüpfungen zwischen Ellen und Reventlow hergestellt. Vor diesem Hintergrund erscheint die Feststellung berechtigt, dass in den Rezensionen zu Ellen Olestjerne das Geschlecht der Autorin im Allgemeinen keine Rolle spielt. Die zuvor zitierte Bemerkung, dass Reventlow in ihrem Roman ,zu frauenhaft“ sei, um zu theoretisieren, ist ein vereinzeltes Beispiel. Rilke betont ausdrücklich, dass Ellens Geschichte sowohl für „Mädchen“ als auch für ,junge Männer“ von Bedeutung sein kann. Als ein Buch, das den Aufstand der Jugend schildert, ist Ellen Olestjerne auch für die ganze Generation repräsentativ, ohne Unterscheidung in Frauen und Männer. ${ }^{8}$

Diese Trennung zwischen der Protagonistin und der Autorin verschwindet allmählich in den Rezensionen zu den nächsten Romanen und Erzählungen von Reventlow: Von Paul zu Pedro. Amouresken (1912), Herrn Dames Aufzeichnungen oder Begegnungen in einem merkwürdigen Stadtteil (1913), Der Geldkomplex (1916), Das Logierhaus „Zur schwankenden Weltkugel“ (1917). Die Rezensionen zu diesen Texten stammen von KARL STECKER (1917/18: 375), Neues vom Büchertisch aus Velhagen\&Klasings Monatshefte (zit. nach KUBITSCHEK 1998:571f.), M.B.[?] (1916/17:427), FRANZ GRAETZER (1917/18:837-841), ANSELMA HEINE (1911/12:863) und THEODOR HEUSS (1916), Einige Bücher, aus März (zit. nach KUBITSCHEK 1998:570f.). In diesen Besprechungen ist - im Unterschied zu den Rezensionen zum Debütroman die Autorin, die inzwischen zu einer nicht unbekannten Münchener Bohemienne wurde, als eine geschlechtsmarkierte Person stets präsent. Darüber hinaus ist diese Autorin in den meisten von ihnen nicht mehr ein Konstrukt, eine anonyme Frau, sondern sie wird konkret und heißt Franziska zu Reventlow. Diese Fleischwerdung der Autorin ist wie gesagt kein geschlechtsneutraler Prozess. Das weibliche Geschlecht wird in den nach 1912 entstandenen Besprechungen vor allem in den Passagen hervorgehoben, in denen der Stil von Reventlows Prosa charakterisiert wird. So schreibt Stecker über das

$8 \quad$ Wobei die Beschreibung der Sexualität und auch die Frage der (außerehelichen) Mutterschaft genauso gut hätte dazu verleiten können, den Roman als eine frauenspezifische oder sogar ,frauenrechtlerische“ Geschichte aufzufassen. 
Logierhaus: „Für solch ein Büchlein genügt dies leichte Plätschern, das an einen Springbrunnen erinnert, während dem Roman mehr das ruhige Dahinrauschen zwischen Ufern als rechte Tonart ziemt. Ein Springbrunnen kann sehr geistreich sein [...] und in der zuerkannten Gabe des Plauderns liegt gewiß weniger ein Vorwurf als eine Anerkennung.“ (Zit. nach KUBITSCHEK 1998: 572) Der Kritiker assoziiert die Sprache des Textes mit dem Plätschern eines Brunnens, und dann bringt er diese Art und Weise zu sprechen mit dem Geschlecht der Autorin in Verbindung:

Man macht sich von der Gräfin Reventlow unwillkürlich ein bestimmtes Bild nach ihrer Art zu plaudern: man denkt sie sich im Kaffeehaus oder in der Flurhalle eines großen Hotels sitzen, die Zigarette in der Hand und sechs bis acht Zuhörer der verschiedensten Rassen und Klassen um sich versammelt. Alle lauschen gespannt ihrer anmutig schwebenden Darstellungsweise, die mit zurückhaltender Ruhe die geschliffenen Waffen des Witzes und einer feinen Bosheit spielen läßt. (Zit. nach KUBITSCHEK 1998:571)

Der Springbrunnen und eine geistreiche Frau, Gräfin Reventlow, sind beides Bilder, die zum Zweck der Charakteristik der Sprache konstruiert werden. Sie sollen den Stil von Reventlow nicht abwertend etwa als weibliches Geplapper abtun, das Ziel der Rezension ist vielmehr, die Assoziationen zu Weiblichkeit als ein positives Charakteristikum auszuspielen. Zwar bleibt die seriöse Prosa, die ,zwischen den Ufern dahinrauscht“, weiterhin wertvoller als das Plätschern des Springbrunnens, aber eine gewisse Meisterschaft im leichten Erzählen wird immerhin als ein Wert an sich angesehen. Ein ähnliches Bild der Autorin, das ebenfalls zur Charakterisierung des Sprachstils herangezogen wird, findet man im Aufsatz Die Humoristin von GRAETZER (1917/18:837) im Literarischen Echo:

Sie [Reventlow - J.G.] rückt den bequemsten aller erreichbaren Klubsessel vor irgendeinen Sektor aus dem Erdgetriebe, ruht aus von (Gottes) Mühsal der Weltschöpfung und prüft die vollendete lorgnettierend, aber nicht aus eitler Altjüngferlichkeit, sondern weil das wieder einen Anlaß zu behaglichem Kichern über die eigene, allzu menschliche Unzulänglichkeit hergibt. Sie sinnt, nur einen Augenblick. Dann faßt sie den Griffel und strichelt auf Papier, was vorliegt.

Auch zu diesem Bild gehören Leichtigkeit und eine gewisse Lässigkeit - ihr Schaffen findet in einem bequemen Klubsessel so nebenbei statt. Dieses Bild lässt wieder an Salon und Konversation denken. Die Assoziation mit salonhaftem, weiblichem Plaudern wird durch die Assoziation mit der französischen Leichtigkeit des Sprechens und des Lebens verstärkt. GRAETZER (1917/18: 839) stellt fest: „Wie sie plaudert, das wird sie selbst kaum mit einem schalen Fremdwortersatz für das gutgallische ,mondain“ gekennzeichnet wissen 
Justyna Górny

wollen. Dem vollendeten Weltmann stellt sie - als Erste - die vollendete Weltfrau gegenüber, die wieder nicht in ihrem tiefsten Wesen ergriffen wäre, wollte man sie nur als Dame einführen."9 Das Wort „mondain“ verweist darauf, was als französische Sprech- und Lebensweise fungiert, und es heißt hier (aber auch in anderen Rezensionen) nicht nur leicht und weiblich, sondern auch verführerisch. ${ }^{10}$ Die französisch anmutende Koketterie zeichne sich auch dadurch aus, dass sie die Grenzen des guten Geschmacks nie überschreite. Diese assoziative Charakteristik des Reventlowschen Stils legt nahe, dass die Sprache ihrer Prosa keine geschriebene, sondern eine gesprochene ist. Die Kritikerinnen und Kritiker empfinden die Lektüre des Textes als ein Gespräch mit einer Frau, deren Bild vor ihren Augen schwebt und die sie mit sich kokettieren lassen (möchten). Sehr deutlich tritt dies auch in der Rezension von Anselma Heine (1911/12:863) zu Von Paul zu Pedro zum Vorschein: „Man sieht das elegante kluge Persönchen, das diese Briefe schreibt, leibhaftig vor sich, erlebt an ihm Überraschungen, hat amüsante und ernstere Stunden, ganz wie mit einer lebenden Person, man läßt sich von ihrem reizvollen Überspiel von Korrektheit zu demimondänster Freiheit verwirren und entzücken. “11 In dieser Passage kann man, genauso wie in der Rezension von Graetzer, sehen, welche Bedeutung die Personifizierung der erzählenden Instanz hat: Wenn sie als (bzw. ganz wie) eine lebende Person betrachtet wird, dann wird sie kein geschlechtlich unmarkierter ,Autor', kein geschlechtsloses Wesen, sondern eine Frau, von der man sich auch noch gerne verführen lässt. Die Lektüre ihrer

9 Die Beschreibung des Nationalcharakters der Franzosen als ,weiblich“ ist ein Thema für sich (FLORACK 2000). Geschlechterklischees und nationale Stereotypen sind miteinander verschränkt. Es sei nur an den Spruch von Jean Paul erinnert, der meinte: „Weiber sind wie Franzosen geborene Weltleute“ - wodurch eben die Leichtigkeit und brillante Oberflächlichkeit beider Gruppen unterstrichen werden sollte.

10 „Der Vergleich mit den französischen Plauderinnen des achtzehnten Jahrhunderts liegt nahe, wenn man diese Briefe einer leichtherzig und weise Liebenden liest, aber sie sind nicht etwa Nachahmungen, sie sind modern und subjektiv trotz des glatten Stils.“ (HeINE 1911/12:863) Vgl. auch: „Gleichmäßig ist in diesen Büchern etwas, was sonst die Franzosen für sich ausschließlich in Anspruch nehmen: die Klarheit des Ausdrucks, die Sicherheit der Pointe, die dünne, reine Luft, unter der jede Linie eher silbergrau als kräftig schwarz oder bunt erscheint:“ (M.B.[?] 1916/17:427).

11 Es muss darauf hingewiesen werden, dass Heine im Unterschied zu Stecker und Graetzer die so charakterisierte Schreiberin der Briefe an keiner Stelle mit Reventlow selbst identifiziert. 
Texte wird als Genuss bereitend beschrieben. Es scheint, dass dieser Genuss einem Spiel entspringt, das zwischen dem in Gestalt der geistreichen Frau verkörperten Text und dem Leser stattfindet. Den Kritikerinnen und Kritikern nach lohnt es sich anscheinend, sich auf dieses Spiel einzulassen.

Das Geschlecht der Autorin ist als ein Teil der Biographie anzusehen, und deshalb sind die Bezüge zu ihrer, Weiblichkeit' als Bezüge zu ihrer Biographie zu betrachten - in den Rezensionen erscheint der Text selbst nicht weniger weiblich als seine Autorin. Die Lektüre als ein leichter, beinahe erotischer Genuss bedeutet nicht nur Begegnung mit dem Text, sondern auch Begegnung mit der Autorin, die ja oft mit ihrem Namen, Reventlow, benannt wird. Die Art und Weise, auf die die Prosa von Reventlow in diesen Rezensionen wahrgenommen wird, ist also sehr stark an das biographische Faktum, dass sie eine Frau ist, gebunden. ${ }^{12}$ Im Unterschied zu den Rezensionen zu Ellen Olestjerne erscheint in den Besprechungen zu späteren Romanen das Geschlecht als ein wichtiger Faktor. Dies führte zu Veränderungen in der Rezeption, die man durchaus als Einschränkungen einstufen kann: War Ellen Olestjerne eine universelle Vertreterin einer ganzen Jugendbewegung, so konnte der Geldkomplex nicht mehr in so einem breiten Kontext gelesen werden - der Briefroman wurde nicht etwa als Satire auf die Psychoanalyse, sondern als die nächste genussvolle Begegnung mit der plaudernden Autorin aufgefasst, ähnlich wie Von Paul zu Pedro oder Herrn Dames Aufzeichnungen. Denn alle Romane und Erzählungen, die nach Ellen Olestjerne entstanden, wurden wie ein Textkorpus gelesen: Der Bezug zu ,Weiblichkeit ' ließ die Unterschiede zwischen den einzelnen Texten verschwimmen. Trotzdem lassen die Rezensionen von Stecker, Graetzer oder Heine immerhin einen Spielraum zu, in dem ihre Prosa als Kunst des Wortes gelesen werden kann; z. B. bedeuten die Vergleiche mit der französischen Leichtigkeit für die Kritik auch die Anknüpfung an eine französische Prosatradition. Graetzer führt in seinem Aufsatz diese Salon-Assoziation weiter und bringt Reventlows Stil in Verbindung mit der romantischen Ironie. Au-

12 Diese Lesart kommt auch in der neueren Sekundärliteratur zum Vorschein. LuDmila Kaloyanova-Slavova (1998) betrachtet den Roman Ellen Olestjerne von Reventlow als Quelle zur Erforschung der kulturellen und geistigen Situation der künstlerisch tätigen Frauen gegen Ende des 19. Jahrhunderts. Die Voraussetzungen, von denen Kaloyanova-Slavova ausgeht, sind gewiss andere - ihr geht es darum, die nicht überlieferte Geschichte der Frauen zu rekonstruieren. Nichtsdestotrotz wird die Literarizität der Reventlowschen Prosa so gut wie gar nicht berücksichtigt. Eine ähnliche Vorgehensweise findet sich auch bei CZARNECKA (2004). Es bleibt allerdings ein Geheimnis, warum im letztgenannten Buch der Name Reventlow konsequent als ,Reventhlow' geschrieben wird. 
Justyna Górny

Berdem wird das Bild der Autorin als einer Frau voller Esprit als Mittel zur Charakteristik ihrer Texte herangezogen, d.h. die Literarizität der Texte bleibt dank der Reflexion über die Sprache mindestens teilweise erhalten.

Die Verbindung zwischen den Texten und der Biographie von Reventlow in der literaturkritischen Wahrnehmung wurde noch enger, nachdem Reventlows Tagebücher erschienen waren. 1925 gab ELSE REVENTLOW Gesammelte Werke in einem Bande von Franziska zu Reventlow heraus, in dem der Fragment gebliebene Roman Selbstmordverein und Tagebücher der Autorin veröffentlicht wurden. In der Rezension von OTTO FlAKE (1926:197-211), der diesen Band bespricht, werden das Leben und die Prosa von Reventlow zu einer Einheit. Was sie verbindet, ist das Geschlecht: „Sie ist interessant vom Anfang bis zum Ende, als Mensch wie als Künstlerin, als Chaos wie als Ordnung, und immer ist sie Frau, Geschlechtlichkeit war hier Schicksal." Die ,Weiblichkeit‘ wird als Grundprinzip des Lebens und des Schaffens von Reventlow aufgefasst. Damit ist aber nicht gemeint, dass dies etwa das Thema ihres Schaffens war, sondern, dass sie sozusagen aus ihrer ,Weiblichkeit ' heraus schrieb: Sie sei eine Frau und schreibe als Frau. Wobei in Flakes Rezension die Unmittelbarkeit dieser zwei Ausdrucksweisen so intensiv betont wird, dass man eher von ,Frau sein“ und ,Frau schreiben“ sprechen sollte. „Sie ist Frau, mehr als sich kann sie nicht gestalten, alles ist Ichform. Höchstens von Ansätzen der Objektivierung darf man sprechen, in denen sich eine merkwürdige, spukhafte, an E.T.A. Hoffmann angenäherte, jedoch weniger düstere, weniger kräftige Stimmung ankündigt; vielleicht wäre sie bei längerem Leben deutlicher geworden." (FLAKE 1926:205) In dieser Passage kommt deutlich zum Vorschein, dass in Flakes Rezension die Texte von Reventlow als eine Art Widerspiegelung oder Verdopplung ihres Lebens betrachtet werden. Zugleich wird dieses Leben als eine kunstvoll ausgelebte Darstellung oder Verwirklichung der Weiblichkeit aufgefasst. In seiner Wahrnehmung verschmelzen die Texte und das Leben von Reventlow zu einem schimmernden Ganzen:

Sie tanzt mit Worten ihre Frivolität, die ewigen Verirrungen der Gleichzeitigkeit und jenes Dreiecks, das bei ihr ein Polygon war. Flucht vor der Tiefe, vor dem Auge in Auge mit den Problemen setzt sie in dieser entstilisierten Zeit instand, im besten Stil der klassischen Mondänen über die Vielheit der Liebesformen, über die Komik und Philistrosität der Männer Konversation zu machen. Die Flucht vor der Tragik ist Selbstschutz. Einem Mann würde man diese Furcht, die Untergründe zu öffnen, kaum verzeihen. Aber die Untergründe sind sehr deutlich da. Die Novellistik der Reventlow ist Equilibristik, was für ein Wort, man muß stottern - aber es ist ein gutes Wort, denn es ersetzt die ethische Harmonie durch die statische. (FLAKE 1926:207f.) 
In dieser Passage klingen die Töne, die auch in früheren Rezensionen auftauchten, sehr deutlich an. „Konversation“, „Frivolität“, „klassische Mondäne“ sind Formulierungen, die an die französisch-leicht plaudernde Verführerin erinnern. Bei Flake wird diese Fleischwerdung der Autorin durch die Erwähnung der körperlichen Bewegung, des Tanzes, noch intensiviert. Die in den anderen Rezensionen betonte Leichtigkeit wird auch von Flake wahrgenommen, wobei er sie interessanterweise als eine gezielte Flucht vor der Tragik und Tiefe interpretiert. Deshalb fasst er die Haltung von Reventlow als „Equilibristik“ auf, als den Versuch, in den Texten ähnlich wie im Leben vor dem tiefreichenden, aber tragischen Ernst zu fliehen. Es stellt sich die Frage, ob dieser Ernst, diese Tiefe, diese „Untergründe“ dort zu sehen oder zu fühlen sind, wo bei Reventlow eine Stimmung à la E.T. A. Hoffmann entsteht. Wären das also die Stellen, in denen die „Ansätze der Objektivierung“ zum Vorschein kommen und das Frau-Sein aufhört? In Flakes Auffassung erscheint die Geschlechtlichkeit, sprich: die ,Weiblichkeit' der Autorin als eine Art Inszenierung. Es gibt zwar in seiner Rezension normative Unterscheidungen zwischen Mann und Frau: An einen Autor stellt Flake andere Forderungen als an eine Autorin. Diese Normierung schließt die etwaige Freiwilligkeit oder Beliebigkeit der GenderInszenierung aus. Gleichzeitig jedoch betrachtet er die Weiblichkeit von Reventlow als ein Lebens-Kunstwerk und ein Stück Leben in der Kunst. Gerade weil die Inszenierung so gekonnt sei, lasse sie das Leben und die Texte als eine Art ,Gesamtkunstwerk ' erscheinen. Flakes Rezension zeigt einen wichtigen Unterschied zu den früheren Rezensionen; in ihr ist nämlich der Schwerpunkt von den Texten auf die Biographie verlegt, vielleicht ist die Veröffentlichung der Tagebücher von Reventlow als eine der Ursachen anzusehen. Die literarischen Texte sind vor allem als Belege wichtig, die eine kulturelle Erscheinung namens, Reventlow' zu verstehen erlauben. Die Literarizität ihrer Prosa bleibt unberücksichtigt, die Vergleiche mit Hoffmann gelten nur für die wenigen Stellen, an denen das ,Frau schreiben “ aufhört, an denen die Inszenierung der ,Weiblichkeit" unterbrochen wird.

Die Rezensionen zu den Romanen und Erzählungen von Reventlow zeigen die übliche Vorgehensweise der literarischen Kritik, die sich mit schreibenden Frauen befasste. Die Frage des Geschlechts blieb immer im Kern ihres Interesses, die Literarizität der Texte wurde dagegen nur wenig berücksichtigt - sie sollten vor allem Dokumente der weiblichen Schreibweise sein. Es wäre aber unfair, gerade die Rezensionen zu der Prosa von Reventlow als eine Diskriminierung der weiblichen Texte zu betrachten. Vielmehr waren die Rezensenten bemüht, eine positive Interpretation, die die ,Weiblichkeit' einbezieht, zu entwickeln. Die ausgrenzende Potenz dieser Betrachtungsweise wird erst aus 
Justyna Górny

zeitlichem Abstand bemerkbar: Die Fixierung auf die, Weiblichkeit' der Autorin hatte zur Folge, dass in den Rezensionen keine Interpretationsmuster für Reventlows Texte ausgearbeitet wurden, die dann durch die literaturwissenschaftliche Forschung hätten übernommen oder auch dekonstruiert werden können. Außerdem wurde durch das ständige Hinweisen auf das Geschlecht der Autorin der Schwerpunkt des Interesses auf den biographischen Aspekt verlagert. Die Biographie wiederum wurde nicht etwa als Rohmaterial für ihre Prosa, sondern als deren Inhalt und Wesen aufgefasst, was dazu beitragen konnte, dass sie letztendlich mehr Aufmerksamkeit als die interessanten Texte von Reventlow weckte.

\section{Literatur}

Becker-CANTARINo, BARbara (1985): Leben als Text. Briefe als Ausdrucks- und Verständigungsmittel in der Briefkultur und Literatur des 18. Jahrhunderts. In: GNÜG, Hiltrud / Möhrmann, Renate (eds.): Frauen Literatur Geschichte. Schreibende Frauen vom Mittelalter bis zur Gegenwart. Stuttgart, 83-103.

BRAнM, Отто (1915): Dorothea Schlegel. In: Kritische Schriften. Bd. 2: Literarische Persönlichkeiten aus dem neunzehnten Jahrhundert. Hrsg. von Paul Schlenther. Berlin, 1-20.

CZARneCKa, MirosŁawa (2004): Wieszczki. Rekonstrukcja kobiecej genealogii w historii niemieckiej literatury kobiecej od połowy XIX w. do końca XX wieku. [Seherinnen. Rekonstruktion der weiblichen Genealogie in der Geschichte der deutschen Frauenliteratur von der Mitte des 19. bis zum Ende des 20. Jahrhunderts]. Wrocław.

Deibel, Franz (1905): Dorothea Schlegel als Schriftstellerin im Zusammenhang mit der romantischen Schule. Berlin (=Palaestra 40$)$.

EDEN, WIEBKE (1998): „Das Leben ist ein Narrentanz“: weiblicher Narzißmus und literarische Form im Werk Franziska zu Reventlows. Pfaffenweiler.

Flake, Отto (1926): Schreibende Welt. In: Die Neue Rundschau 2:197-211.

FLORACK, RUTH (ed.) (2000): Nation als Stereotyp. Fremdwahrnehmung und Identität in deutscher und französischer Literatur. Tübingen.

Fritz, Helmut (1980): Die erotische Rebellion. Das Leben der Franziska zu Reventlow. Frankfurt (M.).

GraetZer, FranZ (1917/18): Die Humoristin. In: Das literarische Echo 14:837-841.

HAHN, BARBARA (1988): „Weiber verstehen alles à la lettre. “ Briefkultur im beginnenden 19. Jahrhundert. In: BRINKER-GABLER, GISELA (ed.): Deutsche Literatur von Frauen. Bd. 2. München, 13-27.

Haym, Rudolf (1870): Die romantische Schule. Ein Beitrag zur Geschichte des deutschen Geistes. Berlin. 
Heine, Anselma (1911/12): F. zu Reventlow, Von Paul zu Pedro. In: Das literarische Echo 15:863.

Heydebrand, RenAte (ed.) (1998): Kanon, Macht, Kultur: theoretische und soziale Aspekte ästhetischer Kanonbildungen. Stuttgart.

Jost, Vera (2002): Fliegen oder Fallen. Prostitution in Literatur von Frauen im 20. Jahrhundert. Königstein/Ts.

Kaloyanova-Slavova, Ludmila (1998): Übergangsgeschöpfe. Gabriele Reuter, Hedwig Dohm, Helene Böhlau und Franziska zu Reventlow. New York.

KuBITSCHEK, BRigitTA (1998): Franziska Gräfin zu Reventlows Leben und Werk. Eine Biographie und Auswahl zentraler Texte von und über Franziska Gräfin zu Reventlow. München/Wien.

M.B.[?] (1916/17): F. Gräfin zu Reventlow. In: Zeitschrift für Bücherfreunde 8/1: 426-478.

ReVentlow, FranZISKA ZU (1925): Gesammelte Werke in einem Band. Hrsg. von Else Reventlow. München.

- (2004): Sämtliche Werke in fünf Bänden. Hrsg. von Michael Schardt. Oldenburg. S.[?] (1904): Ellen Olestjerne. Eine Lebensgeschichte. In: Frankfurter Zeitung, 10.6.1904:1f. (Erste Morgenausgabe).

SiMONS-STÖCKER, HÄNNY (1906): Franziska Gräfin Reventlow. Ellen Olestjerne. Eine Lebensgeschichte. In: Mutterschutz. Zeitschrift zur Reform der sexuellen Ethik 1:26.

ST.[?] (1904): Das Buch einer Zeitströmung. In: Die Neue Rundschau 15/1:765-767.

SzÉKELY, JoHANNES (1979): Franziska Gräfin zu Reventlow. Leben und Werk. Bonn. 